\title{
Cryptanalysis of White Box DES Implementations ${ }^{\star}$
}

\author{
Louis Goubin, Jean-Michel Masereel, and Michaël Quisquater \\ Versailles St-Quentin-en-Yvelines University \\ 45 avenue des Etats-Unis \\ F-78035 Versailles Cedex \\ \{Louis.Goubin, Jean-Michel. Masereel, Michael .Quisquater\}@uvsq.fr
}

\begin{abstract}
Obfuscation is a method consisting in hiding information of some parts of a computer program. According to the Kerckhoffs principle, a cryptographical algorithm should be kept public while the whole security should rely on the secrecy of the key. In some contexts, source codes are publicly available, while the key should be kept secret; this is the challenge of code obfuscation. This paper deals with the cryptanalysis of such methods of obfuscation applied to the DES. Such methods, called the "naked-DES" and "nonstandard-DES", were proposed by Chow et al. [5] in 2002. Some methods for the cryptanalysis of the "naked-DES" were proposed by Chow et al. [5], Jacob et al. [6], and Link and Neuman [7. In their paper, Link and Neuman 7] proposed another method for the obfuscation of the DES.

In this paper, we propose a general method that applies to all schemes. Moreover, we provide a theoretical analysis. We implemented our method with a $\mathrm{C}$ code and applied it successfully to thousands of obfuscated implementations of DES (both "naked" and "non-standard" DES). In each case, we recovered enough information to be able to invert the function.
\end{abstract}

Keywords: Obfuscation, cryptanalysis, DES, symmetric cryptography, block cipher.

\section{Introduction}

In recent years, the possibility of obfuscating programs has been investigated. From a theoretical point of view, Barak et al. [1] have proven impossibility results for the task of obfuscating computer programs. In particular, it turns out that there exists a family of programs such that: on the one hand each program is non learnable (i.e. its execution does not give any information about its original source code), but on the other hand every obfuscator (i.e. the program producing an obfuscation) fails completely when given any program of this family as input. However it has not been proved that specific instances, particularly cryptographic primitives, are impossible to obfuscate.

\footnotetext{
* This work has been supported in part by the French ANR (Agence Nationale de la Recherche), through the CrySCoE project, and by the région Île-de-France.
} 
In 2002, Chow et al. 45 suggested two different obfuscations, one for the AES, the other for the DES. The AES obfuscation was cryptanalysed by Billet et al. [2]3] in 2004. Chow et al. [5] also mounted an attack on their first DES obfuscation version (called "naked-DES"). Jacob et al. [6] and Link and Neuman [7, proposed two other attacks on the "naked-DES". Here, breaking the "nakedDES" means recovering the secret key.

A second version of DES obfuscation, called "nonstandard-DES", was given by Chow et al. [5]. This "nonstandard-DES" is obtained by obfuscating the usual DES composed with initial and final secret permutations. In this context, breaking such a "nonstandard-DES" implementation means recovering the secret key and the secret initial and final permutations.

Moreover, many industrial actors have developed obfuscated implementations of cryptographic algorithms, in particular for DRM, Pay-TV, and intellectual property protection. (e.g. cloakware [12], retroguard [13], Yguard [14]).

This paper is structured as follows : In Section 2, we give an overview of the obfuscation methods given by Chow et al. and by Link and Neumann. Section 3 is devoted to our attack on the "naked-DES". In Section 4, we adapt our attack to the "non standard" DES. Section 5 is devoted to our implementation of this attack. In Section 6, we compare our attack to the one of Wyseur et al. [11]. Finally, we conclude in Section 7 All proofs are available in the appendices.

\section{DES Obfuscation Methods}

Chow et al. [5] proposed two types of DES obfuscation. The first one, called "naked-DES", produces an usual DES. The second one, called the "nonstandardDES", is a slight modification of the standard DES algorithm. This last version is the one they recommend.

Let us describe the "naked-DES" (see Figure 7). The standard DES is implemented by means of many functions. The first one is an affine function $M_{1}$, which is the composition of the initial permutation, the expansion (slightly modified in order to duplicate all the 32 right bits), and a bit-permutation $\phi_{0}: \mathbb{F}_{2}^{96} \rightarrow \mathbb{F}_{2}^{96}$. The role of $\phi_{0}$ is to send 48 bits to the corresponding S-box entries, the 48 remaining bits being sent randomly to the T-box entries (see Figure 8). Eight of these T-boxes are derived from the eight S-boxes of the DES (see Figure 11), and the four remaining T-boxes are identities (or more generally bit permutations, see Figure $8\left(T_{12}\right)$ ). An affine function $M_{2,1}$ follows the T-boxes. This affine function is the composition of the $\mathrm{P}$ and Xor operation of the standard DES, and a bit-permutation $\phi_{1}$ (see Figure 7). Each of the 16 rounds is the composition of the T-boxes and an affine function $M_{2, i}$. The last round is followed by an affine function $M_{3}$ which is the composition of a selection function, and the final permutation. This function takes for arguments the outputs of the affine function $M_{2,16}$ of the last round and returns the ciphertext (see Figure 7).

We will denote by $A_{i}$, one of these components $\left(T_{i}, M_{1}, M_{2, i}\right.$ or $\left.M_{3}\right)$. The obfuscator program computes numbers of random nonlinear permutations on 


\section{Left or middle bits}

(by-passed bits)

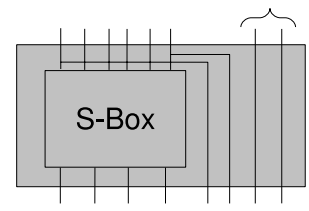

Fig. 1. T-Box

$\mathbb{F}_{2}^{s}, b_{k, l}(s=4$ or 8$)$. These permutations are referred by Chow et al. [5] as io-block encoding bijections. Twenty-four or twelve of these io-block encoding bijections are concatenated in order to obtain nonlinear permutations on $\mathbb{F}_{2}^{96}$, $P_{i, j}$. Each component $A_{i}$ is obfuscated between permutations $P_{i, 1}$ and $P_{i, 2}$. The resulting functions $P_{i, 1} \circ A_{i} \circ P_{i, 2}$ are stored in arrays in order to be used by the obfuscated program. When considering consecutive components, the final permutation of the first component, and the initial permutation of the second component, cancelled out (see Figure 7) i.e. :

$$
\left(P_{i, 1} \circ A_{i} \circ P_{i, 2}\right) \circ\left(P_{j, 1} \circ A_{j} \circ P_{j, 2}\right)=P_{i, 1} \circ\left(A_{i} \circ A_{j}\right) \circ P_{j, 2} .
$$

This "naked-DES" was cryptanalysed by the authors themselves [5].

In order to repair the scheme, they proposed the "nonstandard-DES". It consists in adding two affine bijections $M_{0}$ and $M_{4}$ before and after the "naked-DES", respectively (see Figure (7). It is not specified by Chow et al. [5] whether $M_{0}$ and $M_{4}$ are block encoded (i.e. respectively preceded and followed by nonlinear random permutations). In this paper, we consider that $M_{0}$ and $M_{4}$ are not block encoded.

Further improvement on the attack of the "naked-DES" were given by Link and Neumann [7]. They suggested another solution which consists in merging the T-boxes and the affine function $M_{2, i}$ of each round. This way, we do not have access to the T-boxes outputs. Moreover, the $M_{2, i}$ functions of the different rounds are block encoded in another way.

In this paper, we describe an attack that defeats both "nonstandard-DES" and the Link and Neumann's schemes.

\section{Attack on the "Naked-DES"}

As mentioned before, the "naked-DES" proposed by Chow et al. [5] was already cryptanalysed in the papers [5677. In this section, we show how to cryptanalyse the improved version of the "naked-DES" proposed by Link and Neumann [7. Note that our method also works for the "naked-DES" proposed by Chow et al. [5]. In what follows, we will denote by "regular DES", the one described in the standard 10] (without PC1), and we will use the same notations.

Our attack is divided into two phases and is based on a truncated differential attack. Roughly speaking, the first phase consists in generating pairs of messages 


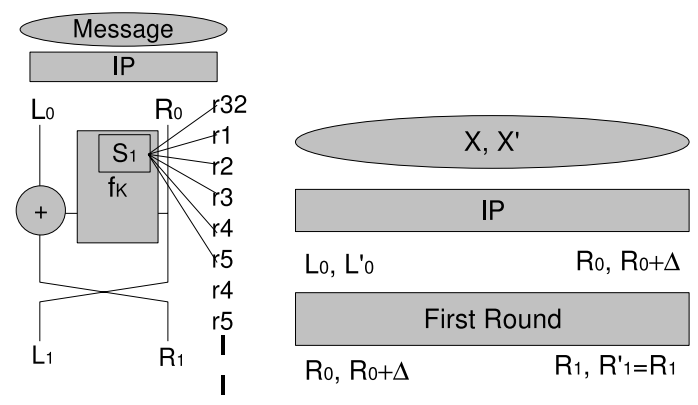

Fig. 2. One round of DES, and attack principle

$\left(X, X^{\prime}\right)$ such that the right part of the images, through $I P$ and the first round of a regular DES, are equal (for a given key $K$ ) (see Figure 2,b). The second phase consists in evaluating those pairs of messages $\left(X, X^{\prime}\right)$ on the "naked-DES" and in checking a condition that we specify below. The pairs that satisfy the test provide a key candidate.

Let us go into the details. Remember that $f(., K)$ denotes the function of the regular DES, we will also denote it by $f_{K}($.$) (see Figure 2$ a). Let $X$ be an initial message, $\left(L_{0}, R_{0}\right)$ denotes its image through $I P$, and $\left(L_{1}, R_{1}\right)$ is the image of $\left(L_{0}, R_{0}\right)$ through the first round, i.e. $\left(L_{1}, R_{1}\right)=\left(R_{0}, L_{0} \oplus f\left(R_{0}, K\right)\right)$. Consider a function $f$, vectors $X$ and $\Delta$, the derivative $f(X) \oplus f(X \oplus \Delta)$ will be denoted by $D_{\Delta} f(X)$. Let us first motivate our algorithm. Let $K$ be a fixed unknown key. Assume we want to find the first round 6-bit subkey corresponding to $S_{i}$ (for the sake of clarity, we will restrain ourselves to $i=1$ ). We generate candidate keys such that only the 6 key bits of $S_{1}$ of the first round are modified. For each of these keys, we compute pairs of messages $\left(X, X^{\prime}\right)$ such that,

1. $\Delta=R_{0} \oplus R_{0}^{\prime}$ is zero, except for the second and third bits.

2. $L_{0}^{\prime}=L_{0} \oplus D_{\Delta} f_{K}\left(R_{0}\right)$

Observe that the second and third bits of $R_{0}$ only affect the output of $S_{1}$ (see Figure 2,a) . Therefore, $f\left(R_{0}, K\right)$ and $f\left(R_{0}^{\prime}, K\right)$ are identical except for the four bits corresponding to the output of $S_{1}$.

Under these conditions, in the next round we have $R_{1}=R_{1}^{\prime}$ and $L_{1}^{\prime}\left(=R_{0}^{\prime}\right)$ is identical to $L_{1}\left(=R_{0}\right)$ except for at most two bits. Consider now these two messages $X$ and $X^{\prime}$ applied to the "naked-DES" with the correct key candidate. We observe that these bits (non-zero bits of $L_{1}^{\prime} \oplus L_{1}$ ) influence at most two io-block encoding bijections $b_{i, 3}$ and $b_{j, 3}$ (see Figure 8). If the key candidate is wrong, we will have $R_{1} \neq R_{1}^{\prime}$. Therefore many bits will change at the output of $M_{2,1}$, and we will be able to distinguish this situation from the correct key guess.

Here is an overview of the attack:

- Randomly choose a message $X$.

- Compute $\left(L_{0}, R_{0}\right)=I P(X)$ with $I P$ public. 
- Choose $\Delta$ such that only the second and third bits are different from 0 .

- For any possible candidate value of 6-bit subkey:

- Compute $L_{0}^{\prime}=L_{0} \oplus D_{\Delta} f_{K}\left(R_{0}\right)$.

- Compute $X^{\prime}=I P^{-1}\left(L_{0}^{\prime}, R_{0} \oplus \Delta\right)$.

- Apply $X$ and $X^{\prime}$ to the obfuscated DES and save the values $Y$ and $Y^{\prime}$ at the end of the first round.

- Compare $Y$ and $Y^{\prime}$ and compute in how many io-block encoding bijections they differ.

- Reject the candidate if this number is strictly greater than 2. Otherwise, the candidate is probably correct.

This way, we can recover the 48 key bits of the first round of the DES. The 8 remaining bits are found by exhaustive search.

Remark 1. This algorithm can produce more than one candidate for the 6-bit subkey. It will provide wrong 6-bit subkeys in two situations.

1. Due to the balance property of the S-boxes and the fact they map six bits to four bits, four different inputs produce the same output. Therefore for each S-box, three wrong 6-bit subkeys will produce the same output as the correct key. To avoid this problem, we can launch this algorithm with another random initial message, or simply another $\Delta$. In fact, we only have to change the values of the bits of $R_{0}$ and $\Delta$ corresponding to the input of $S_{1}$ (the bits $32,1, \ldots, 5)$. Actually, we can choose different pairs $\left(X, X^{\prime}\right)$ such that the intersection of the key candidates associated to each of them is the correct key.

2. The second one is due to a propagation phenomena. Suppose we have a wrong 6-bit subkey producing a wrong $S_{1}$ output. It means that there are more than three bits of difference between $\left(L_{1}, R_{1}\right)$ and $\left(L_{1}^{\prime}, R_{1}^{\prime}\right)$. These differences could be mapped to the same io-block encoding bijection, leading to the flipping of only two io-block encoding bijections at the output of $M_{2,1}$. In this case, we launch this algorithm with several values for $R_{0}$. It leads to several lists of key candidates and the correct key belongs to the intersection. This way, wrong keys will be discarded.

\section{Attack on the "Nonstandard-DES"}

This section is dedicated to an attack on the "nonstandard-DES". Remind that the "nonstandard-DES" is a "naked-DES" where the affine functions $M_{1}$ and $M_{0}$ are replaced by $M_{1} \circ M_{0}$ and $M_{4} \circ M_{3}$ respectively (where $M_{0}$ and $M_{4}$ are mixing bijections, see Chow et al. [5]). As mentioned before, we assume that the inputs of $M_{1} \circ M_{0}$ (respectively the outputs of $M_{4} \circ M_{3}$ ) are not io-block encoded. Note that all the other functions are io-block encoded using bijections on $\mathbb{F}_{2}^{4}$ (the same principle applies for the obfuscation proposed by Link and Neuman [7] where the bijections are defined on $\mathbb{F}_{2}^{8}$ ). Moreover, we assume that the T-Boxes follow the same ordering in the different rounds. In what follows, we will not consider $I P$ (inside $M_{1}$ ) w.l.o.g, for the sake of clarity. 
In what follows, the term preimage will implicitly refer to the preimage with respect to the linear bijection $M_{0}$. Moreover, we say that a bit of a vector is touching an io-block encoding bijection if this bijection depends on this bit. Similarly, we will say that a vector touches an S-Box if non-zero bits touch it.

Our attack on the "nonstandard-DES" is based on the one on the "naked-DES". Our approach is based on a truncated differential attack. It consists in computing the images of a random vector $X_{0}$ at different levels in the obfuscated DES. We compare these values (called initial-entries) to the corresponding images of $X_{0} \oplus$ $\Delta$, where $\Delta$ satisfies some conditions depending on the context. This approach allows providing information about the key and the matrix $M_{0}^{-1}$, gradually. The full key and the matrix $M_{0}^{-1}$ will be known at the end of the process. The way we store information about $M_{0}^{-1}$ consists in considering lists of candidates for preimages of unspecified canonical vectors. Lists of candidates containing only one vector are called distinguished lists. This vector is then a column of $M_{0}^{-1}$. Note that these lists are actually vector spaces and can be shared by several canonical vectors. In practice, a list $E$ will be shared by $\operatorname{dim} E$ canonical vectors (that are not necessary specified). Our algorithm works sequentially and consists in specifying these canonical vectors and shortening the lists. Our method can therefore be understood as a "filtering process". The different filters are described below.

Section 4.1 describes a preliminary step almost independent of the structure of the block cipher. It consists in finding vector spaces associated to a particular ioblock encoding bijection at the input of the first round. This step allows getting global information about $M_{0}^{-1}$.

Section 4.2 describes a set of filters intending to refine information about $M_{0}^{-1}$. These steps are highly related to the studied block cipher. The first filter, described in Section 4.2. allows distinguishing lists that are associated to canonical vectors belonging either to right bits or left bits of the input of the first round $\left(L_{0}\right.$ or $\left.R_{0}\right)$. The second filter, described in Section 4.2 extracts all the lists (marked as "right" in the previous filter) touching a single S-box (we will see that these lists play an important role). The third filter, described in Section 4.2 gathers the lists (marked as "left" in the previous filter) in sets associated to the output of S-boxes. Section 4.2 links T-Boxes (obfuscation of the keyed S-boxes) to S-Boxes. This information allows the last filter, presented in Section 4.2 to precisely specify the 1-to-1 link between the lists (marked as "left") and the (left) canonical vectors.

Section 4.3 explains how to extract the key and how to recover the full invertible matrices $M_{0}^{-1}$ and $M_{4}$.

\subsection{Block Level Analysis of $M_{1} \circ M_{0}$}

Recovering of the $\boldsymbol{B}_{\boldsymbol{k}}$ 's. Denote by $K_{k}$ the space $\left(\{0\}^{4 k-4} \times \mathbb{F}_{2}^{4} \times\{0\}^{96-4 k}\right)$, and by $\bar{K}_{k}$, the space $\left(\mathbb{F}_{2}^{4 k-4} \times\{0\}^{4} \times \mathbb{F}_{2}^{96-4 k}\right)$. In what follows, the vector space spanned by a set of vectors $S$ will be denoted $\langle S\rangle$. Also, $e_{i}$ denotes the ith canonical vector (the position of the "one" is computed from the left and 
start from one) of the vector space $\mathbb{F}_{2}^{64}$. The sets $\left\{e_{i} \in \mathbb{F}_{2}^{64} \mid i=1 \ldots 32\right\}$ and $\left\{e_{i} \in \mathbb{F}_{2}^{64} \mid i=33 \ldots 64\right\}$ will be denoted by $\mathcal{S}_{L}$ and $\mathcal{S}_{R}$, respectively.

Ideally, we are looking for 24 vector spaces such that their vectors influence only one io-block encoding bijection at the output of $M_{1} \circ M_{0}$. This would allow modifying only the input of one particular io-block encoding bijection. Unfortunately, due to the duplication of the bits in $M_{1}$ (because of the expansion $E)$ this goal is impossible to reach. We will therefore try to approximate this situation and deal with the drawbacks afterwards. First we will have to give some notations, definitions and properties.

Denote by $F: \mathbb{F}_{2}^{64} \rightarrow \mathbb{F}_{2}^{96}$ the obfuscation of $M_{1} \circ M_{0}$ (see Figure 7 ).

Let $X$ be a vector in $\mathbb{F}_{2}^{96}$. Denote by $\pi_{k}$ the projection $\pi_{k}:\left(\mathbb{F}_{2}^{4}\right)^{24} \rightarrow \mathbb{F}_{2}^{4}$ : $X=\left(x_{1}, \ldots, x_{24}\right) \mapsto x_{k}$. Let $b_{k}$ be the $k^{\text {th }}$ io-block encoding bijection at the output of $M_{1} \circ M_{0}$. The function $F$ is written as

$F(X)=\left(b_{1} \circ \pi_{1} \circ M_{1} \circ M_{0}(X), b_{2} \circ \pi_{2} \circ M_{1} \circ M_{0}(X), \ldots, b_{24} \circ \pi_{24} \circ M_{1} \circ M_{0}(X)\right)$.

Definition 1. Let $k$ be an integer, $k \in[1,24]$. We denote by $\mathcal{B}_{k}$ the vector space $\left\{X \in \mathbb{F}_{2}^{64} \mid \pi_{k} \circ M_{1}(X)=0\right\}$. In other words, it is the subspace of vector $X$ such that for any non-zero component $e_{i}$ of $X, M_{1}\left(e_{i}\right)$ does not touch $b_{k}$, i.e. $\mathcal{B}_{k}=\left\langle e_{j} \mid \pi_{k} \circ M_{1}\left(e_{j}\right)=0\right\rangle$.

Definition 2. Let $k$ be an integer, $k \in[1,24]$. We denote by $\mathcal{E}_{k}$ the subspace of vector $X$ such that for any non-zero component $e_{i}$ of $X, M_{1}\left(e_{i}\right)$ touches $b_{k}$, i.e. $\mathcal{E}_{k}=\left\langle e_{j} \mid \pi_{k} \circ M_{1}\left(e_{j}\right) \neq 0\right\rangle$.

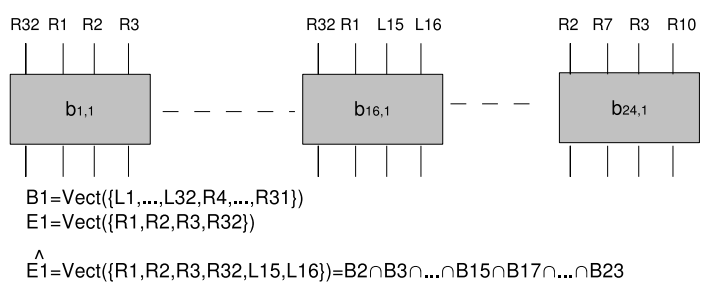

Fig. 3. Example

Remark 2. Note that $\mathbb{F}_{2}^{64}$ is the direct sum of $\mathcal{B}_{k}$ and $\mathcal{E}_{k}$ for any $k$, i.e. $\mathbb{F}_{2}^{64}=$ $\mathcal{B}_{k} \oplus \mathcal{E}_{k}$.

We will denote by $B_{k}$ the subspace $M_{0}^{-1}\left(\mathcal{B}_{k}\right)$, and by $E_{k}$ the subspace $M_{0}^{-1}\left(\mathcal{E}_{k}\right)$.

Proposition 1. For any $k$ integer, $k \in[1,24], B_{k}=\left\{\Delta \in \mathbb{F}_{2}^{64} \mid D_{\Delta} F\left(\mathbb{F}_{2}^{64}\right) \subset\right.$ $\left.\bar{K}_{k}\right\}$, the probability that $\Delta$ belongs to $B_{k}$, when $\Delta$ is randomly chosen, is greater or equal to $\frac{1}{2^{4}}=\frac{1}{16}$, and $60 \leq \operatorname{dim}\left(B_{k}\right)<64$.

Combining Definition 2 and Property 1, the vector space $E_{k}$ can be described as the set of vectors $\Delta$ such that for any vector $X_{0} \in \mathbb{F}_{2}^{64}, M_{0}\left(X_{0}\right) \oplus M_{0}\left(X_{0} \oplus \Delta\right)$ has in total at most four non-zero components $e_{i}$, all of them touching the $k^{\text {th }}$ 
io-block encoding bijection through $M_{1}$. Due to Property 1 it is easier to recover a basis for $B_{k}$ 's, than for $E_{k}$ 's. That is why we will first recover all the $B_{k}$ 's. Using Property 1, we only have to compute $D_{\Delta} F\left(X_{0}\right)$ for random $\Delta \in \mathbb{F}_{2}^{64}$ and determine to which space $\bar{K}_{k}$ it belongs. Using $B_{k}$ 's, we will recover $E_{k}$ 's, or at least, 24 vector spaces $\widehat{E}_{k}$ containing $E_{k}$ with minimal dimension.

Recovering of the $\widehat{\boldsymbol{E}}_{\boldsymbol{k}}$ 's. Let us now explain how to recover $\widehat{E}_{k}$. First, let us remark that for any $X \in \mathbb{F}_{2}^{64}$ and for any $\Delta \in \mathbb{F}_{2}^{64}$, we have $D_{\Delta} F(X) \in \bar{K}_{k}$ if and only if $D_{\Delta} \pi_{k} \circ M_{1} \circ M_{0}(X) \in \bar{K}_{k}$. Let us introduce the following lemma.

Lemma 1. Let $k$ be an integer belonging to [1,24]. If $\mathcal{E}_{j} \cap \mathcal{E}_{k}=\{0\}$ for any integer $j$ distinct from $k$ belonging to $[1,24]$, then

$$
\mathcal{E}_{k}=\bigcap_{j \neq k} \mathcal{B}_{j}
$$

Since $M_{0}$ is a bijection, this lemma means that if $\mathcal{E}_{j} \cap \mathcal{E}_{k}=\{0\}$ for any integer $j \in[1,24]$ different from $k$, then $E_{k}=\bigcap_{j \neq k} B_{j}$. Nevertheless, due to the bitduplication, there exist indices $k$ and $j$ such that $\mathcal{E}_{j} \cap \mathcal{E}_{k} \neq\{0\}$ (and then $\left.E_{j} \cap E_{k} \neq\{0\}\right)$. Denote by $J_{k}$ the set $\left\{j \mid \mathcal{E}_{j} \cap \mathcal{E}_{k}=\{0\}\right\}$, by $\widehat{\mathcal{E}}_{k}$ the subspace $\bigcap_{j \in J_{k}} \mathcal{B}_{j}$, and by $\widehat{E}_{k}$ the subspace $\bigcap_{j \in J_{k}} B_{j}$ where $k$ is an integer belonging to $[1,24]$.

Proposition 2. For any integer $k \in[1,24], \mathcal{E}_{k} \subseteq \widehat{\mathcal{E}}_{k}$.

Let us introduce a property that will allow us to give another characterization of $J_{k}$.

Proposition 3. For any integer $i \in[1,24]$ and for any integer $j \in[1,24]$

$$
\operatorname{dim}\left(\mathcal{E}_{i} \cap \mathcal{E}_{j}\right)=64+\operatorname{dim}\left(B_{i} \cap B_{j}\right)-\operatorname{dim}\left(B_{j}\right)-\operatorname{dim}\left(B_{i}\right) .
$$

A straightforward application of this property to the definition of $J_{k}$ leads to $J_{k}=\left\{j \in[1,24] \mid 64=\operatorname{dim}\left(B_{j}\right)+\operatorname{dim}\left(B_{k}\right)-\operatorname{dim}\left(B_{k} \cap B_{j}\right)\right\}$. This characterization will be useful in order to compute $\widehat{E}_{k}$. If $\operatorname{dim}\left(\widehat{E}_{k}\right)+\operatorname{dim}\left(B_{k}\right)>64$ then $E_{k} \subsetneq \widehat{E}_{k}$, and we have found a vector space containing strictly the one we search. Note that when $\operatorname{dim}\left(\widehat{E}_{k}\right)+\operatorname{dim}\left(B_{k}\right)=64, E_{k}=\widehat{E}_{k}$. This case is particularly interesting because it reduces the complexity of the full cryptanalysis.

\subsection{Bit Level Analysis of $M_{0}^{-1}$}

In the previous section, we were looking for differences $\Delta$ associated to a specific io-block encoding bijection. It allowed us to get some information about $M_{0}^{-1}$. In this section, we refine our search and this will allow us to get enough information about $M_{0}^{-1}$ in order to apply our method on the "naked-DES" to "nonstandardDES". Our algorithm works sequentially and consists in a "filtering process". The different filters are described below. 
Search for Candidates for Preimages of Elements Belonging to the Sets $\mathcal{S}_{\boldsymbol{L}}$ and $\mathcal{S}_{\boldsymbol{R}}$. Consider $\Delta$ be an element of $\mathbb{F}_{2}^{64}$ such that $M_{0}(\Delta)=e_{i}$ and $e_{i} \in \mathcal{S}_{L}$. The only non-zero bit of $M_{1} \circ M_{0}(\Delta)$ touches only one io-block encoding bijection (recall that we do not consider $I P$ ). Therefore, $\Delta$ belongs to a single $\widehat{E}_{k}$. Assume now that $\Delta \in \mathbb{F}_{2}^{64}$ such that $M_{0}(\Delta)=e_{i}$ and $e_{i} \in \mathcal{S}_{R}$ then $M_{1} \circ M_{0}(\Delta)$ has exactly two non-zero bits that may touch the same or two distinct io-block encoding bijections or equivalently $\Delta$ belongs to one or two spaces $\widehat{E}_{k}$. In what follows, we will call double an element $\Delta \in \mathbb{F}_{2}^{64}$ such that $M_{0}(\Delta) \in \mathcal{S}_{R}$ and the two non-zero bits of $M_{1} \circ M_{0}(\Delta)$ touch the same io-block encoding bijection. For example, on Figure 8 , the bit $R_{2}$ could be a double, since its two instances are in the input of $T_{1}$. By considering intersections between the spaces $\widehat{E}_{k}$, taken pairwise, we can distinguish preimages of elements of $\mathcal{S}_{R}$ from doubles or preimages of elements of $\mathcal{S}_{L}$.

Note that the intersections between spaces $\widehat{E}_{k}$ taken pairwise provide more information. Indeed, $\widehat{E}_{i} \cap \widehat{E}_{j}$ contains preimages of unknown canonical vectors. In particular, if $\operatorname{dim}\left(\widehat{E}_{i} \cap \widehat{E}_{j}\right)=1$ then $\widehat{E}_{i} \cap \widehat{E}_{j}=\left\langle M_{0}^{-1}\left(e_{k}\right)\right\rangle$ for some $k$. In this case, we already know the preimage of an unknown canonical vector. When $\operatorname{dim}\left(\widehat{E}_{i} \cap \widehat{E}_{j}\right)>1$ we can still take advantage of this fact even if it requires some extra searches.

Recovering Middle Bits. In order to apply our attack presented in Section 3 , we need to exactly know the preimage of canonical vectors touching only a single S-Box of the first round (e.g. Right bits $2,3,6,7,10, \ldots$ ). In what follows, we will refer to such a canonical vector as a middle bit. If a middle bit is not a double, then its two copies touch two different io-block encoding bijections. The first copy is in input of an S-box, leading to at least two bits of difference at the end of the first round of a regular DES, and 4 bits in our case, due to the expansion. The second copy is a by-passed bit (see Figure1), leading to only one bit of difference at the end of the first round. Consider the bold path in Figure 8 starting from $R_{3}$ bit, in order to have a global view. Let us explain how we use this property.

Recall that $X_{0}$ is the initial-vector defined in Section 4 For each difference $\Delta$ belonging to the lists marked as input of the studied T-box, we apply $X_{0} \oplus \Delta$ to the obfuscated DES by making an injection fault. This means that we set the input of this T-box to the initial-entry while we keep the input of the other TBoxes (see Figure 4). We evaluate the number of io-block encoding bijections at

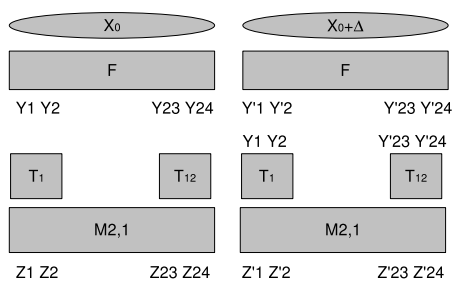

Fig. 4. Injection fault 
the output of the first round that differs from the corresponding initial-entries. If only one io-block encoding bijection (at the output of the first round) differs from the corresponding initial-entry, we deduce that $\Delta$ could be the preimage of a middle bit. Therefore, a list containing preimages of several canonical vectors can be divided into two shorter lists; one list containing preimages of middle bits while the other contains preimages of non-middle bits.

Remark 3. If a T-box is touched by more than three middle bits or left bits, we deduce that this T-box does not contain any S-box. Note also that doubles can only be preimages of middle bits. Finally, a T-box touched by a double contains necessarily an S-box.

Recovering Left Bits. In order to apply our attack presented in Section 3. we need to know which group of four canonical vectors are xored with the output of each S-box of the first round. First, we determine the io-block encoding bijections that are touched by the outputs of the studied S-box and we denote by $B S$ this set of bijections. In Figure 8. we can see that $B S=$ $\left\{b_{1,3}, b_{3,3}, b_{8,3}, b_{12,3}, b_{15,3}, b_{20,3}, b_{24,3}\right\}$ for the S-box $S_{1}$. The elements $b_{i, 3}$ of $B S$ are characterised by $D_{\Delta_{m}} b_{i, 3} \circ \pi_{i} \circ M_{2,1} \circ T \circ M_{1} \circ M_{0}\left(X_{0}\right) \neq 0$, for all $\Delta_{m}$ belonging to a list marked as a middle bit of the studied S-box. Then, we store in an extra list $\mathcal{L}$ each $\Delta$ marked as left bits touching exactly two bijections of $B S$. This list contains all the preimages associated to canonical vectors that are potentially xored with the output of the S-box. Finally, we find $\Delta_{l} \in\langle\mathcal{L}\rangle$ such that for any bijection $b_{i, 3} \in B S$ we have $D_{\Delta_{m} \oplus \Delta_{l}} b_{i, 3} \circ \pi_{i} \circ M_{2,1} \circ T \circ M_{1} \circ M_{0}\left(X_{0}\right)=0$, where $\Delta_{m}$ belongs to a list marked as a middle bit of the studied S-box. This process is repeated with different $\Delta_{m}$ or $X_{0}$, until we find four linearly independent $\Delta_{l}$ or equivalently the vector space spanned by the preimages of the searched canonical vectors. We then compute the intersection between this space and all the lists. It allows us to split some of them in shorter lists (the intersection and the complementary space of the intersection). It may lead to lists containing a single vector (distinguished list).

Chaining. In this section, we will try to determine precisely the correspondence between T-boxes and S-boxes. Due to the remark in Section 4.2, we know which are the T-boxes containing an S-box. The probability that a selected T-box, denoted by $T_{1}$, contains $S_{1}$ is $1 / 8$. We determine the two T-Boxes that are touched by a canonical vector associated to a list marked as "right bit", "nonmiddle bit" and associated to $T_{1}$. Selecting one of these T-Boxes randomly, the

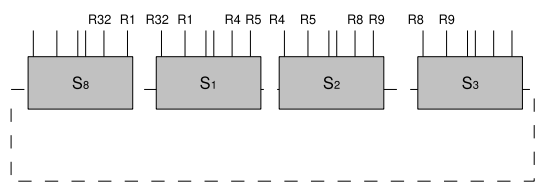

Fig. 5. Chaining 
probability that it contains $S_{2}$ is $1 / 2$. Out of the set of unselected T-Boxes, we select the one that is touched by a canonical vector associated to a list marked as "right bit", "non-middle bit" and associated to the previous selected T-Box. We continue the process until all T-Boxes have been selected (see Figure 5). Note that the probability to determine the right correspondence is $1 / 8 \times 1 / 2=1 / 16$.

Bit Positions. At this stage, we have recovered between others, 32 preimages corresponding to unspecified left canonical vectors. In order to determine the correspondence, we use the following observation on the DES:

\section{Out of the four left bits that are xored with the output of a specified $S$-Box, exactly two become (in the second round) middle bits.}

Now, we just have to apply each of the preimages to the obfuscated DES and check whether the image of this vector in front of the second round is a middle bit (cf.4.2). Assuming that the T-Boxes follow the same ordering in the different rounds, preimages corresponding to a middle bit (resp. non-middle bit) can be distinguished by observing the indices of the touched T-Boxes.

For example, for the first S-box, among the preimages of the four identified left canonical vectors,

- one of such vectors is the preimage of $e_{23}$ (resp. $e_{31}$ ) if it is the preimage of a middle bit of $S_{6}$ (resp. $S_{8}$ ) in the second round.

- one of such vectors is the preimage of $e_{9}$ (resp. $e_{17}$ ) if it is not the preimage of a middle bit and it is in the input of $S_{2}$ and $S_{3}$ (resp. $S_{4}$ and $S_{5}$ ) of the second round.

\subsection{The Attack}

In Section 4.2, we have shown how to recover all the preimages of the left canonical vectors. In other words, we have recovered half of $M_{0}^{-1}$ (columns and their positions). Also, some of the lists marked as middle bits contain only one vector but their corresponding canonical vector is however unknown. Therefore, some columns of $M_{0}^{-1}$ are known up to their positions. Finally, the remaining lists marked as middle bits contain preimages of some canonical vectors $e_{i_{1}}, \ldots, e_{i_{n}}$ (their number is the dimension of the vector space spanned by the list). In this case, we select linearly independent vectors in the list and we associate each of them to one of the canonical vector $e_{i_{j}}$. Therefore, we are in the context of the attack of the "naked-DES" up to some adaptations. In particular, we have to choose $X_{0}$ belonging to the vector space spanned by the known columns of $M_{0}^{-1}$. The evaluation of the first round on $X_{0} \oplus \Delta$ may lead to some difficulties. Indeed, we have to choose $\Delta$ belonging to the preimage of middle bits space which is not necessarily included in the vector space spanned by the known columns of $M_{0}^{-1}$. It turns out that we have to try all the candidates for this part of the matrix $M_{0}^{-1}$. For each of these candidates, we mount an attack like we did on 
the "naked-DES", which provides 48-bit key candidates. Note that wrong keys may be recovered. More importantly, here may be no key for this candidate for this part of the matrix $M_{0}^{-1}$. In other words, it means that we have to discard this candidate.

In order to determine the remaining part of $M_{0}^{-1}$ (columns associated to nonmiddle bits), we apply a similar principle that we used for the "naked-DES". Indeed, we know the key and we know that for the "naked-DES" for any initialmessage $X_{0}$ there always exists a difference $\Delta$ with non-zero right component such that the right part of the differential (evaluated in $X_{0}$ ) of the first round is zero. It means that in the context of the "nonstandard-DES", wrong candidates for $M_{0}^{-1}$ can be discarded. Denote by $K$ the space spanned by the known columns of the candidate for $M_{0}^{-1}$ and by $U$ the unknown columns of the candidate for $M_{0}^{-1}$. We have $K \oplus U=\mathbb{F}_{2}^{64}$. The candidate for $M_{0}^{-1}$ can be discarded if there exists $X_{0} \in K$ such that there does not exist $\Delta$ with a non zero-component in $U$ such that the right part of the differential (evaluated in $X_{0}$ ) is zero.

At this stage, we have a 48-bit key candidate and a candidate for $M_{0}^{-1}$. We make an exhaustive search in order to determine the 8 remaining bits of the key. For each of them we try to solve a linear system in order to find the matrix $M_{4}$. If there is no solution for $M_{4}$ we deduce that the 8-bit key candidate is wrong. If all the 8-bit key candidates are wrong, we discard this particular $M_{0}^{-1}$. Note that this method also works if $M_{4}$ has io-block encoding bijections at its output.

Attack on Link and Neumann obfuscation: Our methods only use the outputs of the first and second round. In particular, we never use the outputs of the Tboxes. Therefore, our two attacks ("naked-DES", and "nonstandard-DES") can be applied on the Link and Neumann [7] obfuscation method. The only difference is that we will deal with larger lists.

\section{Results}

This attack was implemented with a $\mathrm{C}$ code. At each stage of the attack, the number of candidates decreases both for the key and for $M_{0}^{-1}$. Finally, it will lead to a unique 48-bit key candidate, a unique $M_{0}^{-1}$ candidate, and a unique $M_{4}$ candidate. We have tested our attack on thousands of randomly generated

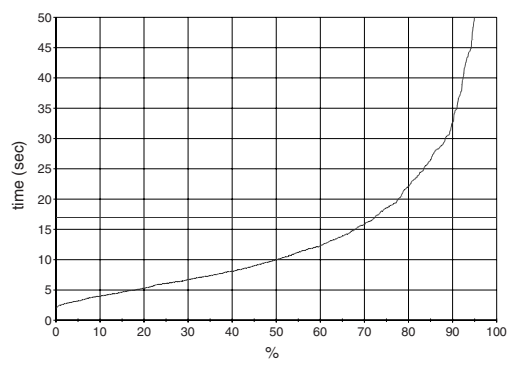

Fig. 6. Repartition of the attacks durations 
obfuscated implementations of DES (both "naked" and "nonstandard" DES). Figure 6 shows the necessary time to complete the attack. We can observe that $95 \%$ of the attacks require less than 50 seconds, and $75 \%$ less than 17 seconds. The mean time is about 17 seconds. However, the attacks were executed on a standard PC. The code was not optimized and the performance can be further improved.

\section{Comparison to Wyseur et al.'s Work}

In this section, we try to clarify the differences between our paper and the one of Wyseur et al. 11. The main advantage of their method is that they are able to recover the key for the "nonstandard-DES" even when the transformations $M_{0}$ and $M_{4}$ are nonlinear. They also briefly explain how to recover these transformations when they are linear, provided the key is known. Our method also allows recovering linear transformations in a short amount of time. The nonlinear case is still an open problem. Finally, Wyseur et al. 11] consider an obfuscation where the $\phi_{i}$ 's have a restricted shape. While our model is unrestricted, they consider only $\phi_{i}$ 's where all middle-bits touch only the four trivial T-boxes. It is not obvious whether their methodology can be adapted to the general case.

\section{Conclusion}

In this paper, we have given new techniques of cryptanalysis for the current obfuscation methods of DES. These techniques rely on a theoretical analysis and have also been implemented as a $\mathrm{C}$ program. We have implemented our method with a $\mathrm{C}$ code and have applied it successfully to more than a thousand obfuscated implementations of DES (both "naked" and "nonstandard" DES). All the studied instances have lead to a unique candidate for the DES key and for the $M_{0}$ and $M_{4}$ secret linear transformations. The key and the two linear transforms have been obtained within 17 seconds in average.

Acknowledgements. The authors would like to thank Sylvie Baudine for proofreading the text.

\section{References}

1. Barak, B., Goldreich, O., Impagliazzo, R., Rudich, S., Sahai, A., Vadhan, S.P., Yang, K.: On the (im)possibility of obfuscating programs. In: Kilian, J. (ed.) CRYPTO 2001. LNCS, vol. 2139, pp. 1-18. Springer, Heidelberg (2001)

2. Billet, O.: Cryptologie Multivariable Ph.D. thesis University of Versailles (December 2005)

3. Billet, O., Gilbert, H., Ech-Chatbi, C.: Cryptanalysis of a white box AES implementation. In: Handschuh, H., Hasan, M.A. (eds.) SAC 2004. LNCS, vol. 3357, pp. 227-240. Springer, Heidelberg (2004) 
4. Chow, S., Eisen, P., Johnson, H., van Oorschot, P.: White-box cryptography and an AES implementation. In: Nyberg, K., Heys, H.M. (eds.) SAC 2002. LNCS, vol. 2595, pp. 250-270. Springer, Heidelberg (2003)

5. Chow, S., Johnson, H., van Oorschot, P., Eisen, P.: A white-box DES implementation for DRM applications. In: Feigenbaum, J. (ed.) DRM 2002. LNCS, vol. 2696, pp. 1-15. Springer, Heidelberg (2003)

6. Jacob, M., Boneh, D., Felten, E.: Attacking an obfuscated cipher by injecting faults. In: Proceedings 2002 ACM Workshop on Digital Rights Management, November 18, 2002, Washington DC, USA (2002)

7. Link, H.E., Neumann, W.D.: Clarifying obfuscation: Improving the security of white-box encoding (2004), http://eprint.iacr.org/

8. Patarin, J., Goubin, L.: Asymmetric cryptography with S-boxes. In: Proc. 1st International Information and Communications Security Conference, pp. 369-380 (1997)

9. Patarin, J., Goubin, L., Courtois, N.: Improved Algorithms for Isomorphisms of Polynomials. In: Nyberg, K. (ed.) EUROCRYPT 1998. LNCS, vol. 1403, pp. 184200. Springer, Heidelberg (1998)

10. http://www.itl.nist.gov/fipspubs/fip46-2.htm

11. Wyseur, B., Michiels, W., Gorissen, P., Preneel, B.: Cryptanalysis of WhiteBox DES Implementations with Arbitrary External Encodings. Cryptology ePrint Archive, Report 2007/104 (2007), http://eprint.iacr.org/

12. http://www.cloakware.com

13. http://www.retrologic.com

14. http://www.yworks.com/en/products_yguard_about.htm

\section{Appendix A: Proofs}

Proof of Property 1: Let $E$ be the set $\left\{\Delta \in \mathbb{F}_{2}^{64} \mid D_{\Delta} F\left(\mathbb{F}_{2}^{64}\right) \subset \bar{K}_{k}\right\}$.

- Let $\Delta$ be an element belonging to $B_{k}$. Let $X$ be an element belonging to $\mathbb{F}_{2}^{64}$.

$$
D_{\Delta} F(X)=\left(D_{\Delta}\left(b_{1} \circ \pi_{1} \circ M_{1} \circ M_{0}(X)\right), \ldots, D_{\Delta}\left(b_{24} \circ \pi_{24} \circ M_{1} \circ M_{0}(X)\right)\right)
$$

According to the definitions, if $\Delta \in B_{k}$ then $M_{0}(\Delta) \in \mathcal{B}_{k}$ or equivalently $\pi_{k} \circ M_{1} \circ M_{0}(\Delta)=0$. Writting $D_{\Delta}\left(b_{k} \circ \pi_{k} \circ M_{1} \circ M_{0}(X)\right)$ as (1), we have :

$$
\begin{aligned}
(1) & =b_{k} \circ \pi_{k} \circ M_{1} \circ M_{0}(X) \oplus b_{k} \circ \pi_{k} \circ M_{1} \circ M_{0}(X \oplus \Delta) \\
& =b_{k} \circ \pi_{k} \circ M_{1} \circ M_{0}(X) \oplus b_{k}\left(\pi_{k} \circ M_{1} \circ M_{0}(X) \oplus \pi_{k} \circ M_{1} \circ M_{0}(\Delta)\right) \\
& =b_{k} \circ \pi_{k} \circ M_{1} \circ M_{0}(X) \oplus b_{k}\left(\pi_{k} \circ M_{1} \circ M_{0}(X) \oplus 0\right) \\
& =b_{k} \circ \pi_{k} \circ M_{1} \circ M_{0}(X) \oplus b_{k} \circ \pi_{k} \circ M_{1} \circ M_{0}(X)=0
\end{aligned}
$$

This means that $D_{\Delta} F(X)$ belongs to $\bar{K}_{k}$ or equivalently $\Delta$ belongs to $E$. We conclude that $B_{k} \subset E$.

- Let $\Delta$ be any element of $E$. According to the definition of $E$, we have in particular $D_{\Delta}(0) \in \bar{K}_{k}$. This means that

$$
b_{k}(0) \oplus b_{k} \circ \pi_{k} \circ M_{1} \circ M_{0}(\Delta)=0,
$$

or equivalently

$$
b_{k}(0)=b_{k} \circ \pi_{k} \circ M_{1} \circ M_{0}(\Delta) .
$$


We deduce that $\pi_{k} \circ M_{1} \circ M_{0}(\Delta)=0$ because $b_{k}$ is a bijection. According to the definitions, it means that $M_{0}(\Delta) \in \mathcal{B}_{k}$ or equivalently $\Delta$ belongs to $B_{k}$. Therefore $E \subset B_{k}$. We conclude that $E=B_{k}$.

- Note that in fact $B_{k}$ is the kernel of $\pi_{k} \circ M_{1} \circ M_{0}$. Since $\operatorname{rank}\left(\pi_{k} \circ M_{1} \circ M_{0}\right)$ is less or equal to 4 , and greater or equal to 1 , we have simultaneously $60 \leq \operatorname{dim}\left(B_{k}\right) \leq 63$ and the probability that $\Delta$ belongs to $B_{k}$ when $\Delta$ is randomly chosen, is equal to $\frac{\operatorname{dim}\left(B_{k}\right)}{2^{64}}$. The results follows.

Proof of Lemma 1; First recall that $\mathcal{B}_{k}=\left\langle e_{j} \mid \pi_{k} \circ M_{1}\left(e_{j}\right)=0\right\rangle$ and $\mathcal{E}_{k}=$ $\left\langle e_{j} \mid \pi_{k} \circ M_{1}\left(e_{j}\right) \neq 0\right\rangle$. Let $\mathrm{j}$ and $\mathrm{k}$ be two distinct integers, then the following conditions are equivalent.

$-\mathcal{E}_{j} \cap \mathcal{E}_{k}=\{0\}$

$-\pi_{k} \circ M_{1}\left(e_{i}\right)=0$ or $\pi_{j} \circ M_{1}\left(e_{i}\right)=0$ for any integer $i \in[1,64]$.

$-\pi_{k} \circ M_{1}(X)=0$ or $\pi_{j} \circ M_{1}(X)=0$ for any vector $X \in \mathbb{F}_{2}^{64}$.

We conclude that if $X \in \mathcal{E}_{j}$ and $\mathcal{E}_{j} \cap \mathcal{E}_{k}=\{0\}$ then $\pi_{k} \circ M_{1}(X)=0$ or equivalently $X \in \mathcal{B}_{k}$.

Consider $X \neq 0$ belonging to $\bigcap_{j \neq k} \mathcal{B}_{j}$. We have that $\pi_{j} \circ M_{1}(X)=0$ for any $j \neq k$. Note that $M_{1}$ is injective. Therefore $M_{1}(X) \neq 0$ and $\pi_{k} \circ M_{1}(X) \neq 0$. We conclude that all the bits of $M_{1}(X)$ that touch $b_{j}(j \neq k)$ are zeros. Therefore, for any non-zero component $e_{i}$ of $X, M_{1}\left(e_{i}\right)$ touches $b_{k}$ or equivalently $X \in \mathcal{E}_{k}$, and $\bigcap_{j \neq k} \mathcal{B}_{j} \subset \mathcal{E}_{k}$.

Let us use an argument by contraposition. Consider $e_{i} \notin \bigcap_{j \neq k} \mathcal{B}_{j}$. Then, there exists $j \neq k$, such that $e_{i} \notin \mathcal{B}_{j}$, i.e. $\pi_{j} \circ M_{1}\left(e_{i}\right) \neq 0$ or equivalently $e_{i} \in \mathcal{E}_{j}$. Therefore, according to the previous three equivalent conditions, $e_{i} \notin \mathcal{E}_{k}$. We deduce that for any $e_{i} \in \mathcal{E}_{k}$ we have $e_{i} \in \bigcap_{j \neq k} \mathcal{B}_{j}$. It means that $\mathcal{E}_{k}=\left\langle e_{i}\right| e_{i} \in$ $\left.\mathcal{E}_{k}\right\rangle \subset \bigcap_{j \neq k} \mathcal{B}_{j}$. We conclude $\mathcal{E}_{k}=\bigcap_{j \neq k} \mathcal{B}_{j}$

Proof of Property 2; Let $e_{i}$ be an element of $\mathcal{E}_{k}$ and $j$ be an element of $J_{k}$. We have $\pi_{k} \circ M_{1}\left(e_{i}\right) \neq 0$ and $\mathcal{E}_{j} \cap \mathcal{E}_{k}=\{0\}$. It implies that $\pi_{j} \circ M_{1}\left(e_{i}\right)=0$, and $e_{i} \in \mathcal{B}_{j}$. Therefore, $e_{i} \in \bigcap_{j \in J_{k}} \mathcal{B}_{j}$, and $\left\langle e_{i} \mid e_{i} \in \mathcal{E}_{k}\right\rangle \subset \widehat{\mathcal{E}}_{k}$.

Proof of Property [3; We will first prove that $\left(\mathcal{B}_{i} \cap \mathcal{B}_{j}\right) \oplus\left\langle\mathcal{E}_{i} \cup \mathcal{E}_{j}\right\rangle=\mathbb{F}_{2}^{64}$.

Consider a canonical vector $e_{k} \notin \mathcal{B}_{i} \cap \mathcal{B}_{j}$. This is equivalent to $\pi_{i} \circ M_{1}\left(e_{k}\right) \neq 0$ or $\pi_{j} \circ M_{1}\left(e_{k}\right) \neq 0$. In other words $e_{k} \in \mathcal{E}_{i}$ or $e_{k} \in \mathcal{E}_{j}$, or equivalently $e_{k} \in\left\langle\mathcal{E}_{i} \cup \mathcal{E}_{j}\right\rangle$. This means that for any canonical vectors $e_{k}$ of $\mathbb{F}_{2}^{64}$, we have either $e_{k}$ belongs to $\mathcal{B}_{i} \cap \mathcal{B}_{j}$ or $e_{k}$ belongs to $\left\langle\mathcal{E}_{i} \cup \mathcal{E}_{j}\right\rangle$.

Assume that there exists a canonical vector $e_{k} \in\left(\mathcal{B}_{i} \cap \mathcal{B}_{j}\right) \cap\left\langle\mathcal{E}_{i} \cup \mathcal{E}_{j}\right\rangle$. We have $\pi_{i} \circ M_{1}\left(e_{k}\right)=\pi_{j} \circ M_{1}\left(e_{k}\right)=0$, and either $\pi_{i} \circ M_{1}\left(e_{k}\right) \neq 0$ or $\pi_{j} \circ M_{1}\left(e_{k}\right) \neq 0$. It leads to a contradiction. Hence $\left(\mathcal{B}_{i} \cap \mathcal{B}_{j}\right) \cap\left\langle\mathcal{E}_{i} \cup \mathcal{E}_{j}\right\rangle$ contains no canonical vectors. 
Assume now that there exists an element $\Delta \in\left(\mathcal{B}_{i} \cap \mathcal{B}_{j}\right) \cap\left\langle\mathcal{E}_{i} \cup \mathcal{E}_{j}\right\rangle$ having a non-zero component $e_{k}$. The vector $\Delta$ belongs to $\left(\mathcal{B}_{i} \cap \mathcal{B}_{j}\right)$, hence $e_{k}$ belongs to $\left(\mathcal{B}_{i} \cap \mathcal{B}_{j}\right)$. Moreover $\Delta$ belongs to $\left\langle\mathcal{E}_{i} \cup \mathcal{E}_{j}\right\rangle$, hence $e_{k}$ belongs to $\left\langle\mathcal{E}_{i} \cup \mathcal{E}_{j}\right\rangle$. Therefore $e_{k}$ belongs to $\left(\mathcal{B}_{i} \cap \mathcal{B}_{j}\right) \cap\left\langle\mathcal{E}_{i} \cup \mathcal{E}_{j}\right\rangle$ which is impossible. We conclude that $\left(\mathcal{B}_{i} \cap \mathcal{B}_{j}\right) \cap\left\langle\mathcal{E}_{i} \cup \mathcal{E}_{j}\right\rangle=\{0\}$. Therefore $\left(\mathcal{B}_{i} \cap \mathcal{B}_{j}\right) \oplus\left\langle\mathcal{E}_{i} \cup \mathcal{E}_{j}\right\rangle=\mathbb{F}_{2}^{64}$.

We deduce that

$$
\begin{aligned}
64 & =\operatorname{dim}\left(\left\langle\mathcal{E}_{i} \cup \mathcal{E}_{j}\right\rangle\right)+\operatorname{dim}\left(\mathcal{B}_{i} \cap \mathcal{B}_{j}\right) \\
& =\operatorname{dim}\left(\mathcal{E}_{i}+\mathcal{E}_{j}\right)+\operatorname{dim}\left(\mathcal{B}_{i} \cap \mathcal{B}_{j}\right) \\
& =\operatorname{dim}\left(\mathcal{E}_{i}\right)+\operatorname{dim}\left(\mathcal{E}_{j}\right)-\operatorname{dim}\left(\mathcal{E}_{i} \cap \mathcal{E}_{j}\right)+\operatorname{dim}\left(\mathcal{B}_{i} \cap \mathcal{B}_{j}\right)
\end{aligned}
$$

Moreover $\mathcal{E}_{i} \oplus \mathcal{B}_{i}=\mathbb{F}_{2}^{64}=\mathcal{E}_{j} \oplus \mathcal{B}_{j}$. Hence $64=64-\operatorname{dim}\left(\mathcal{B}_{i}\right)+64-\operatorname{dim}\left(\mathcal{B}_{j}\right)-$ $\operatorname{dim}\left(\mathcal{E}_{i} \cap \mathcal{E}_{j}\right)+\operatorname{dim}\left(\mathcal{B}_{i} \cap \mathcal{B}_{j}\right)$. The result follows. 


\section{Appendix B: Figures}

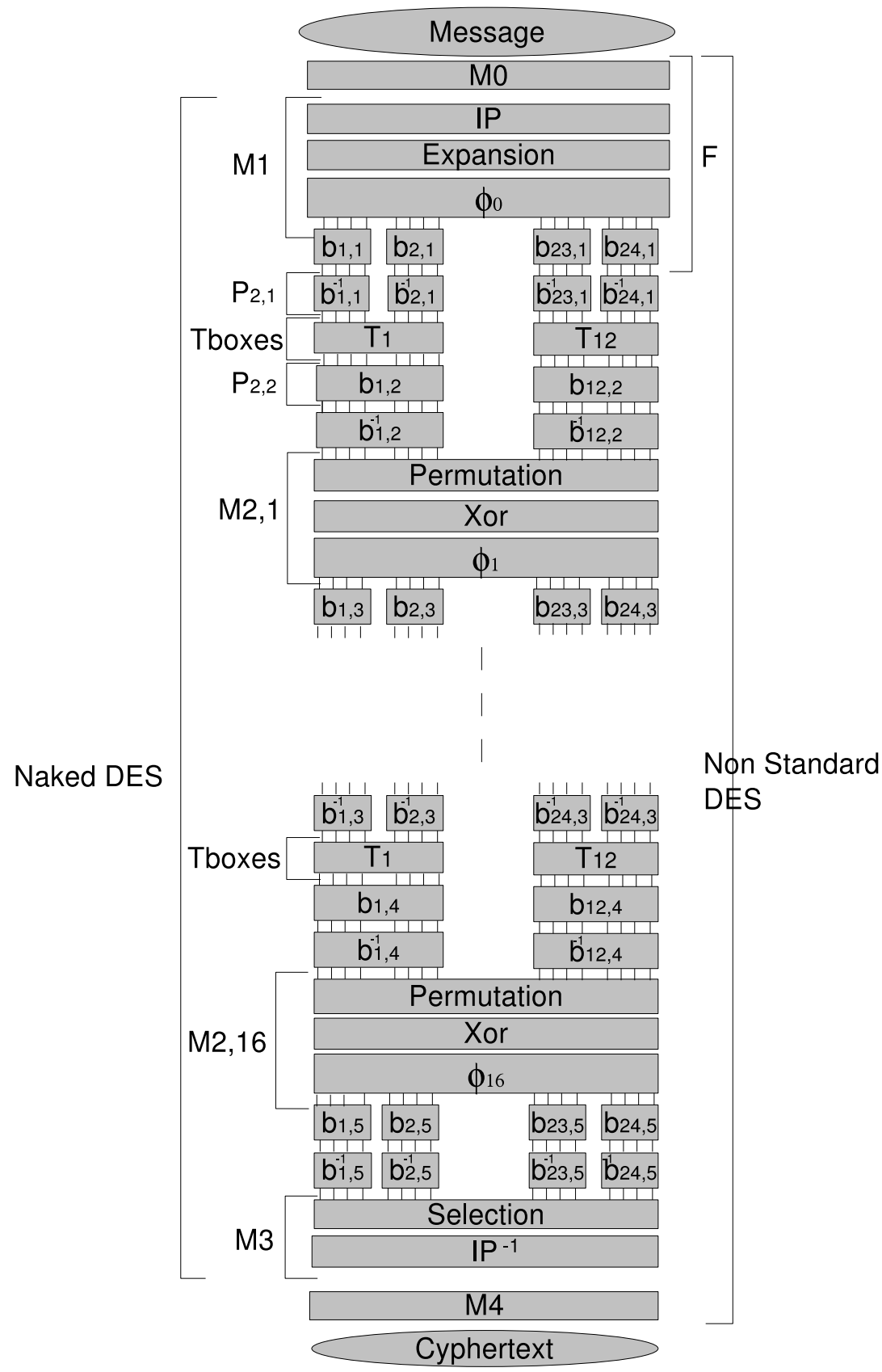

Fig. 7. "Naked-DES" and "Nonstandard-DES" 


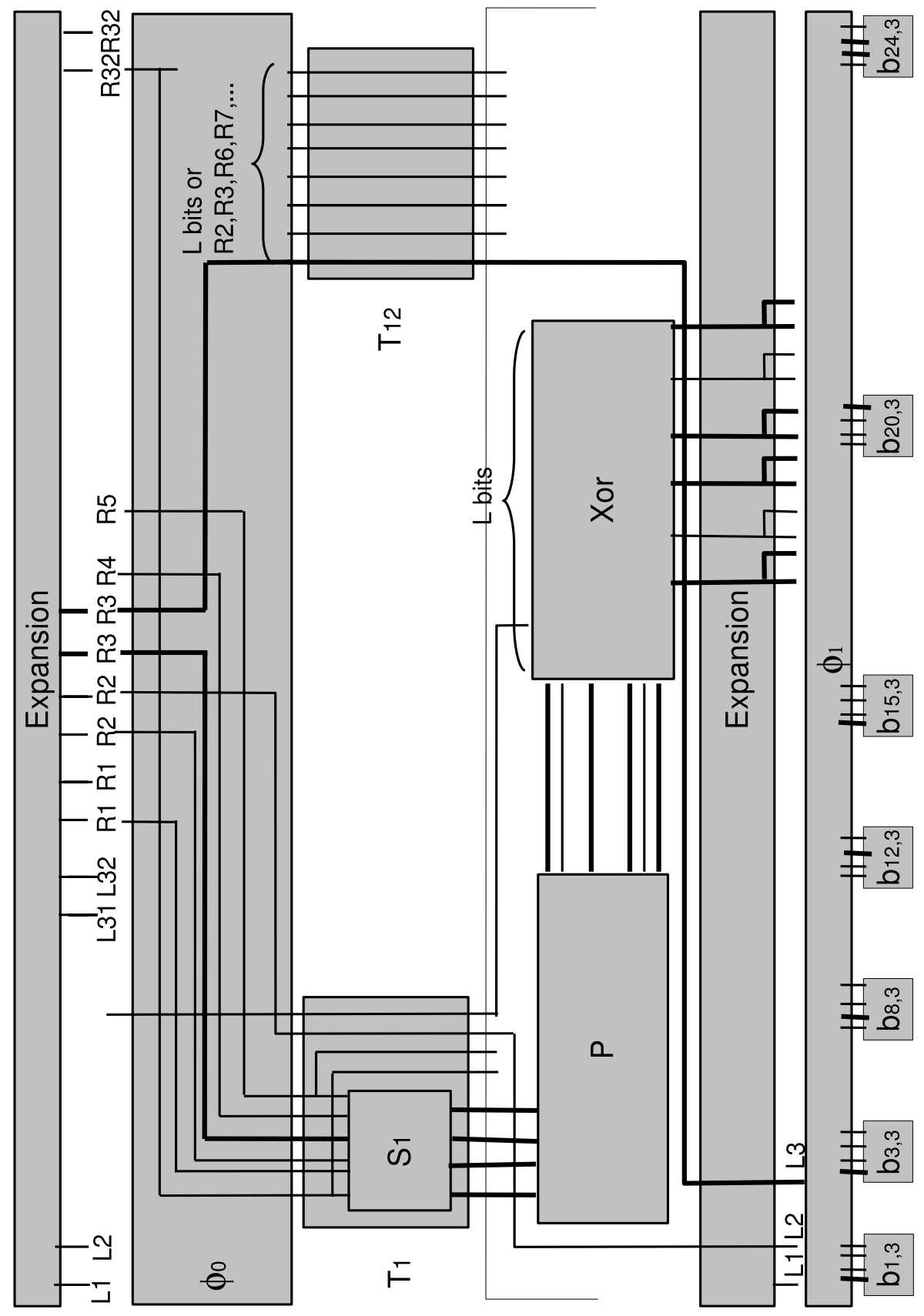

Fig. 8. General view of the attack 